\section{Unintentional inversion of corneal buttons during penetrating keratoplasty: clinico-pathological report of two cases}

${ }^{1}$ Department of Ophthalmology Royal Hallamshire Hospital Sheffield, UK

${ }^{2}$ Ophthalmic Sciences Unit University of Sheffield, UK

\section{${ }^{3}$ Department of Ophthalmology Doncaster Royal Infirmary Doncaster, UK \\ ${ }^{4}$ Cornea Service Pathology Laboratory The Eye Hospital Dortrecht, Rotterdam The Netherlands \\ ${ }^{5}$ Pathology Laboratory The Eye Hospital Dortrecht, Rotterdam The Netherlands \\ Correspondence: \\ $S$ Dinakaran \\ Department of \\ Ophthalmology \\ A-Floor \\ Royal Hallamshire Hospital Glossop Road \\ Sheffield S10 2JF, UK \\ Tel: + 441142711900 \\ Fax: + 441142713682 \\ E-mail: sdinakaran@ \\ yahoo.com}

Received: 22 October 2002 Accepted: 24 January 2003
S Dinakaran'1, MA Parsons², SP Desai WH Beekhuis ${ }^{4}$ and CM Mooy ${ }^{5}$ grafted cornea. Prognosis for repeat penetrating keratoplasty appears to be good. These cases have implications for the understanding of the factors affecting epithelialization of the anterior chamber.

\section{Methods}

This report describes the histopathological features of the corneal grafts that had failed and were replaced in two patients.

Histopathological examination showed that the corneal buttons were inverted, with the epithelial side facing the anterior chamber. The clinical behaviour of these grafts is also described.

Results

Case 1

Clinical features A healthy 27-year-old man underwent penetrating keratoplasty for keratoconus in the left eye in August 1992. He received a $7.5 \mathrm{~mm}$ corneal button obtained fresh from a 23-year-old donor who was HIV and hepatitis B negative. The graft remained clear initially, although he was photophobic from the second postoperative day. In October 1992, the graft became oedematous although he had a visual acuity of $6 / 12$ with pinhole. Progression of the corneal oedema resulted in a visual acuity of only 6/36 with contact lens in December 1992. A diagnosis of primary graft failure was made; at this time, his intraocular pressure and other ocular findings were normal.

He underwent a repeat penetrating keratoplasty in September 1994, when he received a $7.5 \mathrm{~mm}$ corneal button from a matched donor. This second graft was clear at 
his last review 6 years after the second keratoplasty (Figure 1a), except for a small area of opacity adjacent to the host-graft junction in the nasal aspect. His visual acuity with correction was $6 / 12$. On slit-lamp examination, the host corneal endothelium appeared normal, and the intraocular pressure was normal.

Pathology The following description is that of the button removed at the time of repeat keratoplasty. The $7.5 \mathrm{~mm}$ diameter corneal disc had a diffuse slight opacity, and the previous healed keratoplasty scar was visible macroscopically, as the button included a rim of host cornea. Histologically, the peripheral host cornea was oedematous, with oedematous epithelium and stroma at this site. Descemet's membrane here was around $5 \mu \mathrm{m}$ thick, and there were few endothelial cells. The majority of the central part of the graft was donor cornea (Figure $1 b-f)$, which was inverted (inside-out). The external surface of the donor cornea had squamous epithelium of 20 and $120 \mu \mathrm{m}$ thickness (two to eight cells), with a smoothly curved external surface in continuity with the host epithelium. The epithelium on the donor cornea lay directly on Descemet's membrane, which had very prominent undulations and folds. The donor corneal stroma was $600-900 \mu \mathrm{m}$ thick and oedematous, with some collagenous remodelling and a keratoplasty scar, but no cellular infiltrate or vascularization. The internal border of the donor stroma was dense, with characteristics of Bowman's layer.

On the internal surface of the donor cornea there was squamous epithelium (Figure 1c, d) - the original epithelium of the donor cornea, which was viable and 12-60 $\mu \mathrm{m}$ (two to five cells) thick. This epithelium was somewhat attenuated and occasional squamous cells had very large nuclei, up to $18 \mu \mathrm{m}$ in diameter, with prominent eosinophilic inclusions, up to $9 \mu \mathrm{m}$ in diameter, which resembled cytoplasmic intranuclear inclusions. This epithelium extended to the donor-host interface on the internal corneal surface, but did not extend over the endothelial cells of the adjacent host cornea. However, in some areas this epithelium extended to the surgical resection margin probably corresponding to the thin rim of the donor button left behind during the second keratoplasty.

\section{Case 2}

Clinical features A 61-year-old man developed a sterile nonhealing corneal ulcer as a result of radiation keratopathy. He underwent a penetrating keratoplasty in 1995. A 3-mm button from a donor cornea was transplanted to a 3-mm recipient opening, and was covered by a conjunctival flap. The vision postoperatively was $6 / 24$, but after 6 weeks the button was opaque and the wound leaked. As a result, he had a further penetrating keratoplasty (6-mm diameter). The excised cornea was subjected to histopathological examination. The result of the second keratoplasty was good, and 3 years postoperatively there was no evidence of epithelial downgrowth in the anterior chamber, and no signs of failure of the second graft.

Pathology The following description is that of the button removed, which included the 3-mm donor cornea from the first penetrating keratoplasty. Histologically, a 6-mm diameter corneal button showed that the eccentrically placed 3-mm diameter sutured button was inverted (Figure 1g), as the internal surface was covered with epithelium and the external surface with Descemet's membrane (with no overgrowth from the host epithelium). On the internal surface of the cornea, there was no extension of donor epithelium over the host endothelium (Figure $1 \mathrm{~g}$ and $\mathrm{h}$ ). There was focal host epithelial ingrowth at the donor/host interface (not shown), which was partially obliterated by fibrous and a mixed cellular inflammatory infiltrate. The host endothelium was atrophic and the epithelium showed changes of bullous keratopathy.

\section{Discussion}

These two patients underwent unintentional inverse keratoplasty for the treatment of keratoconus and radiation keratopathy, respectively. To our knowledge, there is only one previous single case report of inverse keratoplasty in humans in the literature. ${ }^{1}$ It is possible that the condition might not have been recognized clinically or pathologically.

Following the inverse keratoplasty, epithelium of the host cornea grew over the external surface of the donor graft in patient 1, covering Descemet's membrane. There were no surviving donor endothelial cells, and it is likely that these were lost very soon after keratoplasty.

Patient 2, however, had no epithelial migration on Descemet's membrane, although it is possible that this occurred but that the epithelium was subsequently lost. Ohlrich's patient ${ }^{1}$ however had epithelial overgrowth of Descemet's membrane 8 days postoperatively. Our second patient's host corneal epithelium was affected by irradiation keratopathy, which may have modified its regenerative capacity under these unusual circumstances.

The donor epithelium remained viable (although somewhat attenuated in patient 1 ) on the internal corneal 
b

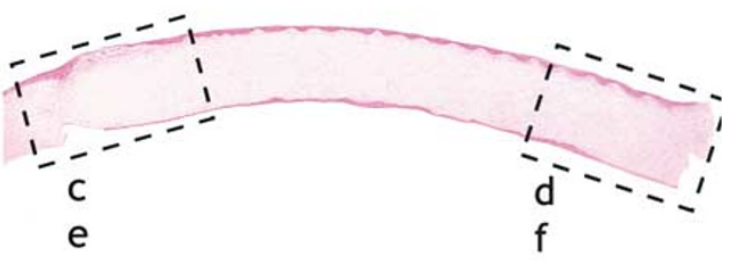

d

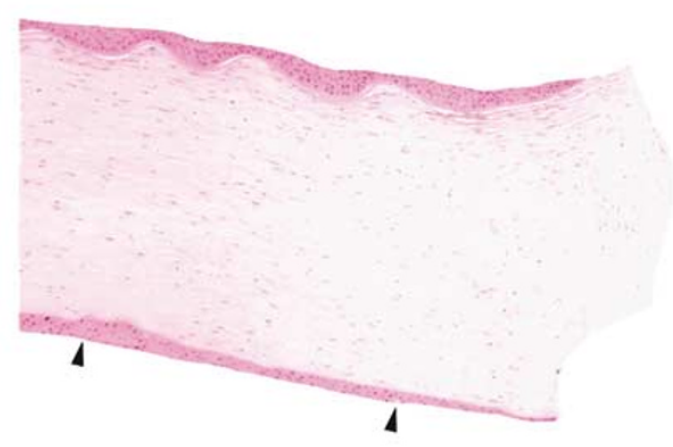

f

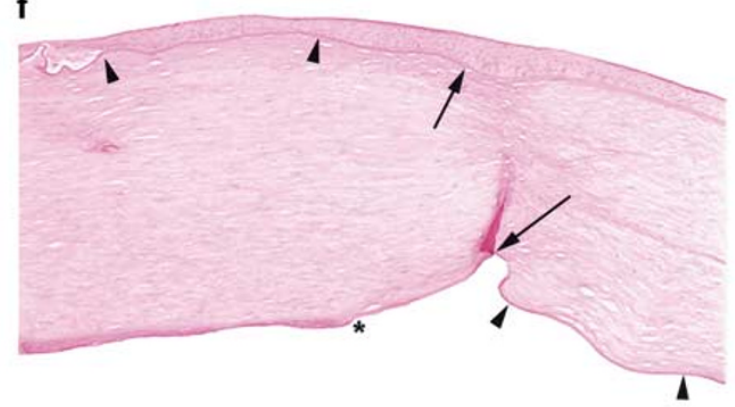

h
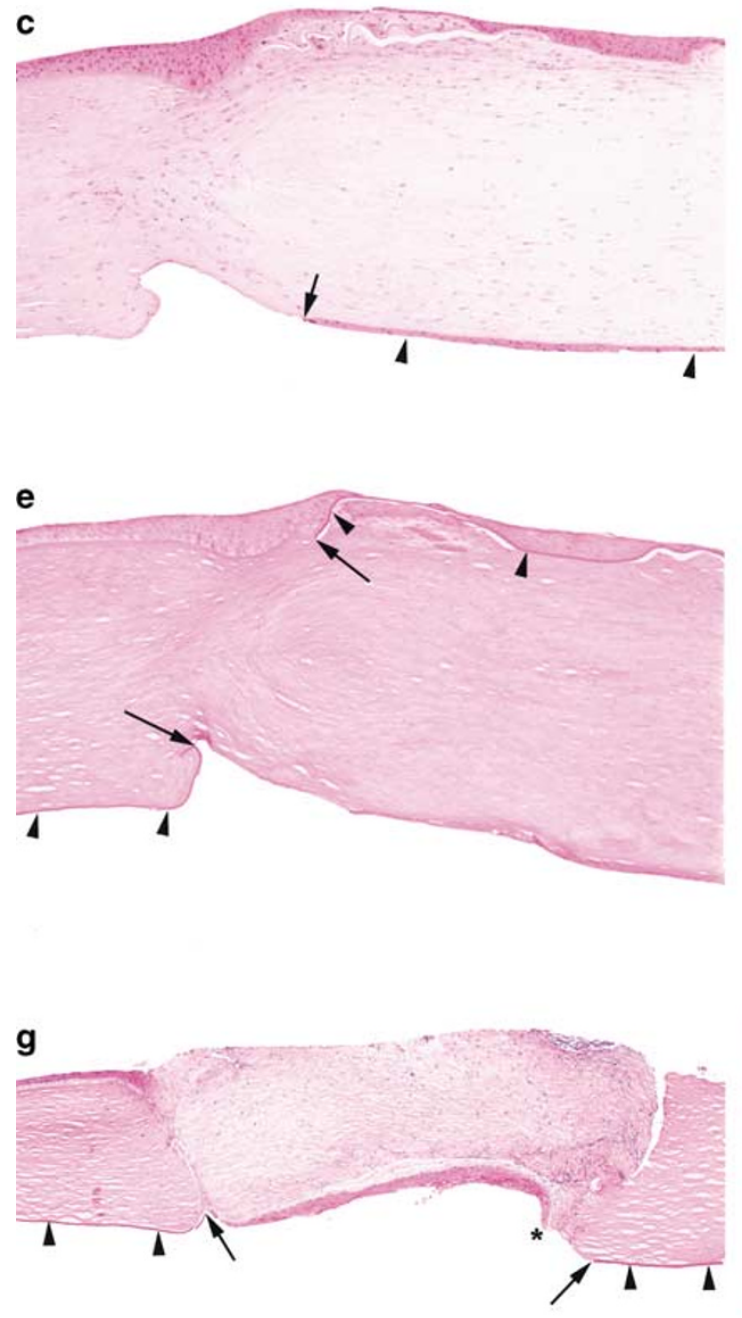

e
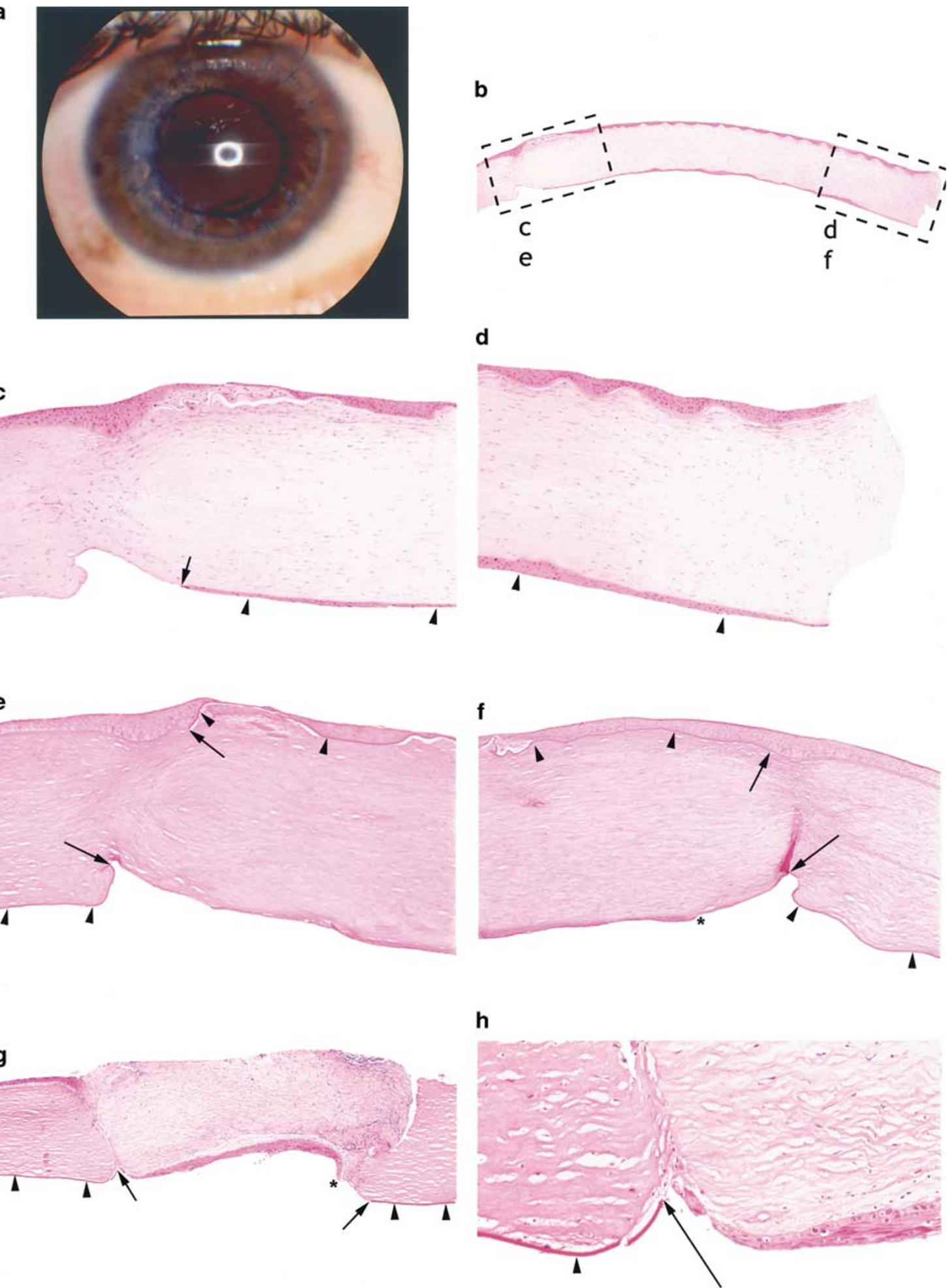

Figure 1 Clinical and pathological appearances of the cornea in patient 1 (a-f) and patient 2 ( $g$ and $h$ ) (a) Cornea of patient 1 , after the second graft. (b) Low-power histology of the cornea, showing the external (top) and internal surfaces (inset sites c-f) (H\&E, magnification $\times 15)$. Boxed areas indicate sites in photomicrographs c-f. (c, d) Detail of the corneal host-graft interfaces. The donor epithelium on the internal surface (arrowheads) is attenuated, and its interface with host Descemet's membrane is arrowed (H\&E, magnification $\times 25)$. $(\mathrm{e}, \mathrm{f})$ PAS stain (magnification $\times 25)$ demonstrates Descemet's membrane (arrowheads) and the ends of Descemet's membrane (arrows) and epithelium $\left(^{*}\right)$. (g) The cornea of patient 2 (H\&E, magnification $\times 20$ ) has the inverted central graft (external surface top), with no central epithelium. Descemet's membrane (arrowheads) extends to the arrow. There is epithelium on the internal surface extending from the left arrow to the asterisk $\left(^{*}\right)$. (h) Enlargement (H\&E, magnification $\left.\times 60\right)$ of the interface (arrow) between host Descemet's membrane (arrowhead) and the epithelium on the internal surface of the graft. 
surface in our two cases and in Ohlrich's patient. ${ }^{1}$ Interestingly, this epithelium remained localized on the donor cornea with no epithelialization of the adjacent internal surface of the host cornea. Surprisingly, gross corneal decompensation did not appear to have occurred in patient 1 , whose cornea remained relatively clear for around 2 years. Decompensation appears to have occurred by around 6 weeks in patient 2 , although this was hard to assess in view of the inflammation and wound leakage, which had been present at this stage. Ohlrich's patient ${ }^{1}$ had a relatively clear cornea at 8 days, although some inflammation (but no wound leakage) was present. None of the three patients had any histological evidence of immunological graft rejection. The donor epithelium extended to some of the internal corneal surgical resection margins in patient 1 , suggesting that some epithelium remained focally near the first keratoplasty scar in this patient. However, there was no clinical evidence of epithelialization of the anterior chamber beyond the edges of the inverted donor corneal tissue 6 years after the second keratoplasty in patient 1 , after 3 years in patient 2 , or in Ohlrich's case ${ }^{1}$ after 9 months.

It is perhaps somewhat surprising that the donor corneal epithelium, present on the internal surface of the cornea, remained viable in all of these patients (for a maximum of 2 years in patient 1 ) between the original and the subsequent keratoplasty without cellular immunological graft failure. It is difficult to hypothesize how an immunological rejection would have manifested. In addition, there is no evidence to suggest that this internally situated epithelium has grown beyond the original confines of the edge of the donor cornea, to cause epithelialization of the anterior chamber angle. This may be because of relative hypoxia, suggested by Arkin ${ }^{2}$ to inhibit epithelial ingrowth in experimental reverse keratoplasty.

All components of the cornea - the epithelium, stroma, and endothelium - are important in wound healing. In some malpositioned corneal wounds, the host corneal epithelium may proliferate into the wound, and as a result of downgrowth may eventually come into contact with the endothelium. There is some evidence from short-term experiments on explanted corneal buttons that there is contact inhibition of epithelial cells by endothelial cells under such circumstances, ${ }^{3,4}$ although Zagorski et $a l^{5}$ demonstrated that this inhibition was overcome in tissue culture systems after 4 weeks. Autologous inverse keratoplasty has been used experimentally to study corneal epithelial-endothelial cell interactions, ${ }^{2,6,7}$ and in these experiments epithelialization of the whole anterior chamber did not occur, as a result of inhibition by fibrin clotting and stromal proliferation. ${ }^{6,7}$ Epithelial proliferation occurs, however, in the presence of extensive anterior synechiae. 8,9

In their study of inverse keratoplasty in rabbits, Zagorski et $a l^{7}$ showed that fibrin clot on the epithelial and wound surfaces facing the anterior chamber suppressed epithelial cell growth in the anterior chamber and formed a matrix for rapidly dividing stromal fibroblasts, while stimulating their growth. Under these circumstances, the inverted epithelium was overgrown by stromal fibroblasts and eventually disappeared; this fibroblastic proliferation ceased after tissue had been covered by corneal endothelium. They suggested that fibrin clotting and stromal proliferation are important factors in preventing epithelial cell growth on the inner surface of the cornea.

In the two cases described, it is possible that fibrin in the anterior chamber was an initial factor preventing epithelial proliferation over the internal surface of the cornea, but there was no evidence of stromal fibroblastic proliferation playing a significant role in epithelial cell growth inhibition at this site. There was no evidence of fibroblastic proliferation causing the disappearance of this epithelium, as was suggested by Zagorski's et al's study. ${ }^{7}$ There was no evidence of immunological rejection of the donor corneal tissue - had this been present, its effect on the growth of 'foreign' epithelium might well have been inhibitory. It remains possible that relative hypoxia inhibited growth of the epithelium, as suggested by Arkin, ${ }^{2}$ but whether there are other mechanisms remains open to speculation.

In reporting patient 1 , we raise what is probably only a slight possibility that epithelialization of the whole anterior chamber could occur after the second keratoplasty, as the new graft might in theory alter the biological dynamics of the situation. However, the fact that such extensive epithelialization had not occurred from the residual epithelium in the 2 years after the first keratoplasty (this is usually a rapid process) suggests that the prognosis remains favourable in this patient. This patient's cornea was clear after 6 years (as is the situation in patient 2 after 3 years).

Inadvertent inverse keratoplasty should be considered as a rare cause of corneal graft failure, even if the graft remains relatively clear. Fortunately, it appears from three patients that this is unlikely to cause the serious complication of anterior chamber epithelialization with secondary glaucoma. The prognosis following repeat penetrating keratoplasty appears to be very good.

\section{Acknowledgement}

We are grateful to Mr Robin Farr for his work on the illustration graphics. 


\section{References}

1 Ohlrich S, Hirst LW, Harrison M, Green WR, Bancroft BJ Inadvertent corneal button inversion during penetrating keratoplasty. Cornea 1992;11:586-588.

2 Arkin W. On reverse experimental transplantation of corneas (in Polish). Post Okulist 1954; 1: 120-123.

3 Cameron JD, Flaxman BA, Yanoff M. In vitro studies of corneal wound healing: epithelial-endothelial interactions. Invest Ophthalmol Vis Sci 1974; 13: 575-579.

4 Yanoff M, Cameron JD. Human cornea organ cultures: epithelial-endothelial interactions. Invest Ophthalmol Vis Sci 1977; 16: 269-263.

5 Zagorski Z, Holbach L, Hofmann C, Gosslar B, Naumann GOH. Corneal epithelial and endothelial cell proliferation in the trabecular region of human donor eyes in vitro. Ophthalmic Res 1987; 19: 28 (abstract).

6 Zagorski Z, Gossler B, Hofmann C, Naumann GOH Experimentelle inverse autologe Keratoplastik beim Kaninchen. Zentralbl Ges Ophthalmol 1987; 129: 75 (abstract).

7 Zagorski Z, Michelson G, Naumann GOH. Experimental autologous inverse keratoplasty - epithelial, stromal and endothelial interactions. Graefe's Arch Clin Exp Ophthalmol 1990; 228: 55-57.

8 Burries TE, Nordquist RE, Rowsey JJ. Model of epithelial downgrowth. I. Clinical correlations and light microscopy. Cornea 1983; 2: 277-287.

9 Smith DR, Sommerville GM, Shea M. An experimental model of epithelialization of the anterior chamber. Can J Ophthalmol 1967; 2: 158-162. 